\title{
Adherence to oral antineoplastic agents in pediatric oncology. A multicenter study
}

\author{
Débora Farberman, Master ${ }^{a}$, Paloma Valente, B.S. ${ }^{a}$, Luciana Malpiedi, B.S. ${ }^{b}$, \\ Mariana Morosi, B.S. ${ }^{b}$ and Lorenzo Luisella, B.S. ${ }^{c}$ \\ Collaborators: \\ Almendra P., B.S., Carro, J., B.S., Couselo, S., B.S., Estévez, N., B.S., García Cozzi, P., B.S., \\ Ghirardi, S., B.S., Miranda, C., B.S., Moser, M., B.S., Otarola, S., B.S., Pérez, S., B.S., \\ Salvia, L., M.D., and Sibelli, C., B.S.
}

\begin{abstract}
Objective. To determine the adherence to oral maintenance medication among pediatric cancer patients and know their beliefs about medications.

Population and methods. Information was obtained from parents, adolescents, and oncologists from six public children's hospitals of Argentina during 2018 and 2019. Questionnaires on adherence (Simplified Medication Adherence Questionnaire) and beliefs about medication (Beliefs About Medicines Questionnaire) were administered. Patients were considered adherent if they referred taking their medication without missing a dose and complying with fasting time. Results. N = 203 patients. Parent-reported adherence was $75 \%$; adherence estimated by oncologists, $82 \%$; and that referred by adolescents, $45 \%$. The outcome measures associated with adherence were financial solvency, diagnosis, treating hospital, number of children under the care of the caregiver, cancer treatments received, and presence of barriers in compliance. Sample subjects did not perceive medications as harmful, but considered they were overused. Most subjects perceived the necessity to take antineoplastic agents to maintain or recover their health, but also expressed their concerns. The perception about medication was similar between parents and adolescents, and was not associated with the level of adherence. Conclusions. In this sample, parent-reported adherence was $75 \%$. Parents and patients perceived a necessity for medication and expressed their concerns about treatment.

Key words: treatment compliance and adherence, leukemia, maintenance, pediatrics.
\end{abstract}

http: / / dx.doi.org/10.5546/ aap.2021.eng.44

To cite: Farberman D, Valente P, Malpiedi L, Morosi M, Luisella L. Adherence to oral antineoplastic agents in pediatric oncology. A multicenter study. Arch Argent Pediatr 2021;119(1):44-50.

\section{GLOSSARY}

ALL: acute lymphoblastic leukemia. AML: acute myeloid leukemia. BMQ: Beliefs About Medicines Questionnaire.

LL: lymphoblastic lymphoma.

PL: promyelocytic leukemia.

SMAQ: Simplified Medication Adherence Questionnaire.

\section{INTRODUCTION}

In Argentina, approximately 1300 pediatric patients (0-15 years old) are diagnosed with cancer every year. Their survival rate 5 years after diagnosis is $70-80 \%$ in international facilities. In our country, it is $60-70 \%{ }^{1}$

A prolonged antineoplastic treatment with oral chemotherapy maintenance helped to reduce recurrence and mortality. Given that healing chances decrease when maintenance compliance is below $95 \%$, achieving an optimal adherence is critical. The administration of maintenance medication is the responsibility of patients / caregivers at home. According to the international bibliography, adherence in pediatric oncology is 8-95\%.3,4

Disease-treatment-facility, patientfamily, and cultural characteristics may act as barriers. ${ }^{5,6}$ People's beliefs about medications may affect adherence. ${ }^{7}$ They carry a major cultural component and warn about the inconvenience of extrapolating adherence barriers from one population to another. The objective of this study was to determine the adherence to oral maintenance medication among pediatric cancer patients and know their beliefs about medications. 


\section{POPULATION AND METHODS}

Six public hospitals participated in the study. The coordinating facility, Hospital Garrahan, is a national oncology pediatric referral hospital that caters for one third of total patients in Argentina. The other five participating hospitals were located in Buenos Aires, Córdoba, Misiones, Santiago del Estero, and Tucumán. Data were collected in 2018 and 2019.

The sample was purposively selected, and subjects were invited to participate if they met the following inclusion criteria: age 0 to 17 years, diagnosed with acute leukemia or lymphoblastic lymphoma, and receiving maintenance with oral antineoplastic agents for at least 30 days. Subjects receiving palliative care and adolescents with cognitive impairment that prevented them from understanding questionnaires were excluded.

Information was obtained from parents, patients older than 13 years, and treating oncologists. Nine of the 18 staff oncologists were asked about their perception on adherence to medication by each patient that was included in the sample. Adherence was assessed using the Simplified Medication Adherence Questionnaire (SMAQ) and operationalized as "adherent" vs. "non-adherent." Adherent patients were defined as those who referred taking their antineoplastic medication without missing a dose and complying with fasting time or consulting immediately after making a change (change in schedule, dose interruption due to the presence of symptoms, etc.). Information about sociodemographic characteristics and potential adherence barriers was obtained.

The Beliefs About Medicines Questionnaire $(B M Q)^{7}$ was administered to parents and patients older than 13 years to determine their beliefs about medications. This tool organized information into four domains. "Harm" and "overuse" assessed the perception about medications in general. "Harm" referred to the damage medications may cause on health, whereas "overuse" indicated their excessive use.

The "necessity" and "concern" domains assessed the perception about the specific treatment medication they were taking at the moment; for this reason, items included the patient's name and the drugs they received. "Necessity" referred to the importance given to medications in relation to health recovery or maintenance. "Concern" asked about associated doubts and fears.

The study was assessed by the Ethics Committee of each participating site. The
Research Ethics Review Committee of Hospital Garrahan approved the project on 12-4-2017. Parents and adolescents signed an informed consent/assent.

Analyses were done based on frequencies and central tendency and dispersion measures, as applicable. Comparisons were done using contingency tables and the $\chi^{2}$ test, with a statistical significance level of less than 0.05 . The statistical software package used was Stata 12.0.

\section{RESULTS}

At study initiation, 237 subjects met the eligibility criteria. A total of 215 protocols were administered due to recurrence, death, new diagnoses, patient referral to a facility with a higher level of care, and difficulties to contact the subject. Five protocols were eliminated, either because the patient was not receiving treatment or had recurrence when completing the questionnaires, had started maintenance treatment recently, or did not answer several items. Seven protocols, from Misiones and Tucumán, were administered weeks after the data collection period had ended and were not included in the database. The resulting sample was made up of 203 subjects. Half of participants were from Hospital Garrahan and the other half, from the remaining facilities.

Parent-reported adherence was $75 \%$. The main reason for non-adherence was forgetfulness. Adolescent-reported adherence was $45 \%$. Oncologists estimated adherence at $82 \%$.

Table 1 shows the main sociodemographic characteristics. The distribution of diagnoses was consistent with the prevalence of such conditions in this population. Mothers were mostly the main caregiver. Almost half of patients (46\%) had to migrate to receive treatment. Access to medication was easy for most patients, but $26 \%$ mentioned difficulties to obtain it.

Practically all parents (98\%) referred that it was easy for them to understand the treating physicians' indications. In the case of adolescents, this value was somewhat lower but still high: $86 \%$.

Five percent of patients had a comorbidity prior to cancer diagnosis, and $16 \%$ acquired a permanent or prolonged comorbidity due to antineoplastic treatment. Among parents, $30 \%$ referred oral medication adverse events; $37 \%$ of them were moderate to severe.

Fourteen percent of parents stated that the difficulties to obtain the medication were a barrier in treatment compliance. The following 
outcome measures showed an association with adherence: financial solvency (having enough money to afford disease-related expenses), number of children under the care of the caregiver, diagnosis, treatment facility, number of cancer treatments, and barriers in treatment compliance. In this sample, beliefs and concerns about disease and treatment were not associated with differences in adherence (Table 2).

Having met basic needs (according to the National Statistics and Censuses Institute of Argentina, INDEC), the level of education of the main caregiver, having social and spiritual support, the frequency of hospital controls, receiving treatment in one or more facilities, migrating, and previous and acquired comorbidities were not associated with differences in adherence in our sample of patients (Figure 1). Experiencing oral medication adverse events was not associated with the level of adherence as reported by parents, but with that reported by adolescents, which was $11 \%$ lower among those who reported symptoms. The small subgroup size prevented us from generalizing such difference.

TABLE 1. Sample description. $N=203$

\begin{tabular}{|c|c|c|}
\hline Outcome measure & Category & $\mathbf{N}(\%)$ \\
\hline Age & $\begin{array}{l}0 \text { to } 2 \text { years old } \\
2 \text { to } 6 \text { years old } \\
6 \text { to } 11 \text { years old } \\
11 \text { to } 13 \text { years old } \\
13 \text { to } 15 \text { years old } \\
15 \text { to } 18 \text { years old }\end{array}$ & $\begin{array}{l}5(2.46) \\
70(34.48) \\
73(35.96) \\
18(8.87) \\
21(10.34) \\
16(7.88)\end{array}$ \\
\hline Treatment facility & $\begin{array}{l}\text { Garrahan } \\
\text { Sma. Trinidad } \\
\text { Niño Jesús } \\
\text { C. Gianantonio } \\
\text { Eva Perón } \\
\text { F. Barreyro }\end{array}$ & $\begin{array}{l}112(55.2) \\
25(12.3) \\
19(9.4) \\
7(3.4) \\
18(8.9) \\
22(10.8)\end{array}$ \\
\hline Diagnosis & $\begin{array}{l}\text { ALL } \\
\text { LL } \\
\text { PL } \\
\text { AML }\end{array}$ & $\begin{array}{l}163(80.3) \\
8(3.9) \\
13(6.4) \\
19(9.4)\end{array}$ \\
\hline Place of residence & $\begin{array}{l}\text { CABA } \\
\text { Greater Buenos Aires } \\
\text { Buenos Aires }>150 \mathrm{~km} \\
\text { Other provinces } \\
\text { Foreigners }\end{array}$ & $\begin{array}{l}10(4.9) \\
66(32.5) \\
9(4.4) \\
113(55.7) \\
5(2.5)\end{array}$ \\
\hline Basic needs & $\begin{array}{l}\text { Met } \\
\text { Unmet }\end{array}$ & $\begin{array}{l}153(75.4) \\
50(24.6)\end{array}$ \\
\hline Enough money to afford treatment & $\begin{array}{l}\text { Enough } \\
\text { Not enough } \\
\text { Did not answer }\end{array}$ & $\begin{array}{l}85(41.9) \\
111(54.7) \\
7(3.4)\end{array}$ \\
\hline Caregiver's level of education & $\begin{array}{l}\text { Primary education } \\
\text { Secondary education } \\
\text { Tertiary/university education }\end{array}$ & $\begin{array}{l}52(25.7) \\
107(52.7) \\
44(21.7)\end{array}$ \\
\hline No. of children under the caregiver's care & $\begin{array}{l}\text { One (patient) } \\
\text { Up to three } \\
\text { More than three }\end{array}$ & $\begin{array}{l}64(31.5) \\
104(51.2) \\
35(17.2)\end{array}$ \\
\hline
\end{tabular}

Treatment facility: a. Hospital Nacional de Pediatría Prof. Dr. Juan P. Garrahan. b. Hospital Santísima Trinidad, Córdoba. c. Hospital del Niño Jesús, Tucumán. d. Hospital Materno-Infantil Dr. C. Gianantonio, San Isidro, Buenos Aires. e. Hospital CePSI Eva Perón, Santiago del Estero. f. Hospital Pediátrico Dr. F. Barreyro, Posadas, Misiones. Diagnosis: ALL (acute lymphoblastic leukemia), LL (lymphoblastic lymphoma), PL (promyelocytic leukemia), AML (acute myeloid leukemia).

Basic needs: as per INDEC's criteria (valid for 2018).

Enough money to afford treatment: at the patient's discretion.

No. of children under the caregiver's care: underage children living in the same household and dependent on the main caregiver. 


\section{Beliefs about medications}

In this sample, most patients did not attribute a harmful effect to allopathic medications. Agreement with "Medicines do more harm than good" and "Natural remedies are safer than medicines" was below $10 \%$, and only $2 \%$ of patients used complementary therapies. The perception of medication overuse by physicians was higher, with a high level of agreement $(70 \%)$ with the statement "Doctors place too much trust on medicines" (Table 3).

In this sample, $90 \%$ of parents agreed with “(Patient name)'s health, at present, depends on (name of antineoplastic agent)" and "(Medication) prevents (patient name)'s (leukemia or lymphoma) from worsening." All items in this domain evidenced that subjects perceived a necessity for antineoplastic agents.

As described by half of the sample subjects, the concerns with the higher level of agreement were "I sometimes worry about the long-term effects of (name of antineoplastic agent)" and
"(Name of antineoplastic agent) is a mystery to me." Considering all items, the score for concern was, however, low (Table 3). Parents' and adolescents' perception, especially in relation to antineoplastic agents, was similar (Figure 2).

\section{DISCUSSION}

The $75 \%$ adherence reported by parents accounted for an intermediate value compared to the Hispanic population of Memphis, USA $(80-85 \%)^{2}$ and higher than that reported in Tampa, USA (60-65\%), ${ }^{8}$ Boston, USA (59 \%), ${ }^{9}$ and developing countries. ${ }^{3,4,10}$ Consistent with the bibliography, adolescents reported a low level of adherence. ${ }^{3,11-13}$

Differences in diagnosis, treating hospital, economic situation, children under the caregiver's care, number of treatments received, and the presence of barriers in compliance were associated with discrepancies in adherence. In some cases, differences were not statistically significant, probably due to the small subgroup

TABLE 2. Adherence and barriers $I . N=196$

\begin{tabular}{|c|c|c|c|c|}
\hline Outcome measure & Category & Adherent, N (\%) & Non-adherent, N (\%) & $p$ value \\
\hline Treatment facility & $\begin{array}{l}\text { Garrahan } \\
\text { Sma. Trinidad } \\
\text { Niño Jesús } \\
\text { Gianantonio } \\
\text { CePSI Eva Perón } \\
\text { Fernando Barreyro }\end{array}$ & $\begin{array}{c}91(81.3) \\
14(58.3) \\
11(73.3) \\
6(85.7) \\
14(77.8) \\
12(60)\end{array}$ & $\begin{array}{l}21(18.8) \\
10(41.7) \\
4(26.7) \\
1(14.3) \\
4(22.2) \\
8(40)\end{array}$ & 0.151 \\
\hline No. of children & $\begin{array}{l}1 \text { (patient) } \\
\text { Up to } 3 \\
\text { More than } 3\end{array}$ & $\begin{array}{l}51(83.6) \\
75(73.5) \\
22(66.7)\end{array}$ & $\begin{array}{l}10(16.4) \\
27(26.5) \\
11(33.3)\end{array}$ & 0.051 \\
\hline No. of cancer treatments & $\begin{array}{l}1 \\
2\end{array}$ & $\begin{array}{c}141(76.2) \\
7(63.6)\end{array}$ & $\begin{array}{c}44(23.8) \\
4(36.4)\end{array}$ & 0.346 \\
\hline Enough money to afford treatment & $\begin{array}{l}\text { Yes } \\
\text { No }\end{array}$ & $\begin{array}{l}69(81.2) \\
79(71.2)\end{array}$ & $\begin{array}{l}16(18.8) \\
32(28.8)\end{array}$ & 0.106 \\
\hline Diagnosis & $\begin{array}{l}\text { ALL } \\
\text { AML } \\
\text { LL } \\
\text { PL }\end{array}$ & $\begin{array}{c}119(75.3) \\
17(94.4) \\
5(71.4) \\
7(53.8)\end{array}$ & $\begin{array}{c}39(24.7) \\
1(5.6) \\
2(28.6) \\
6(46.2)\end{array}$ & 0.077 \\
\hline Barriers in compliance with indications & $\begin{array}{l}\text { Yes } \\
\text { No }\end{array}$ & $\begin{array}{c}12(44.4) \\
136(80.5)\end{array}$ & $\begin{array}{l}15(55.6) \\
33(19.5)\end{array}$ & 0.000 \\
\hline
\end{tabular}

$\mathrm{N}$ refers to the information provided by parents.

Treatment facility: Hospital Nacional de Pediatría Prof. Dr. Juan P. Garrahan, Hospital de Niños de la Santísima Trinidad (Córdoba), Hospital del Niño Jesús (Tucumán), Hospital Municipal Materno-Infantil de San Isidro Dr. C. Gianantonio (Buenos Aires), Hospital Centro Provincial de Salud Infantil Eva Perón (Santiago del Estero), Hospital Pediátrico Dr. F. Barreyro (Misiones). No. of children under the caregiver's care: underage children living in the same household and financially dependent on the main caregiver.

Number of cancer treatments provided for primary disease, recurrence or second malignancy.

Enough money to afford treatment: at the patient's discretion.

Diagnosis: ALL (acute lymphoblastic leukemia), LL (lymphoblastic lymphoma), PL (promyelocytic leukemia),

AML (acute myeloid leukemia).

Barriers in compliance with indications: dealing with their children's resistance to take their medication and comply with fasting time, difficulties obtaining the medication, and others. 
size of non-adherent patients.

The most common barrier in adherence was dealing with children's refusal (to wake up, to take the medication, and to comply with fasting for 1-2 hours before and after taking it). Other studies pointed out a lack of patient cooperation in daily treatment as an outcome measure that required exploration during office visits and improvement. 3,13

Although diagnosis subgroups were numerically dissimilar and some were very small, adherence based on diagnosis tended to be different in terms of complexity and the duration of each drug regimen. The acute myeloid leukemia (AML) group used a daily agent and showed the highest level of adherence; the promyelocytic leukemia (PL) group used a combination of three agents administered in a different manner on a daily basis and showed the lowest level of adherence, and the acute lymphoblastic leukemia and lymphoblastic lymphoma (ALL and LL) groups used a daily

FIGURE 1. Adherence and barriers II. $N=196$

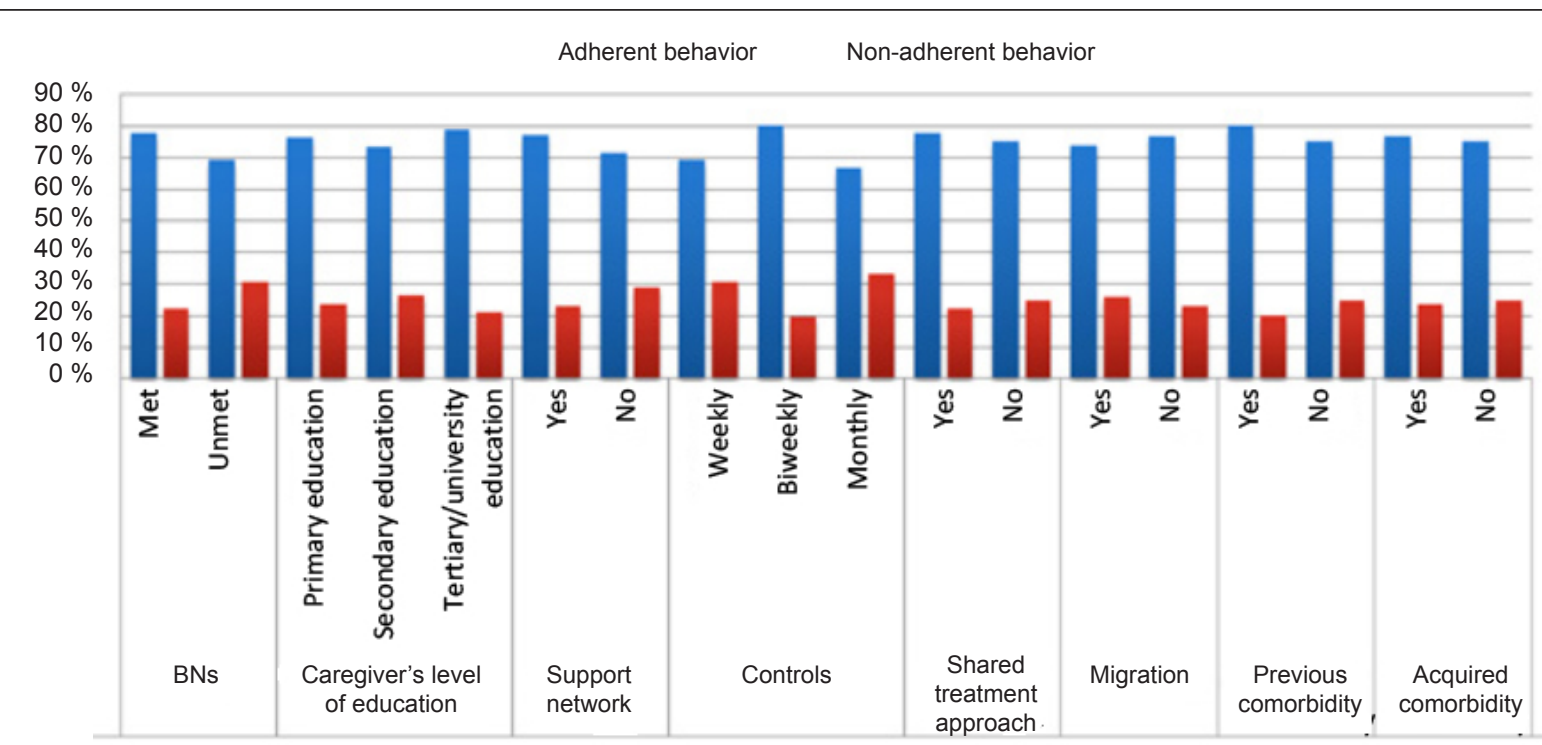

$\mathrm{N}$ refers to the information provided by parents.

Basic needs (BNs): as per INDEC's criteria (valid for 2018).

Support network: family, friend, and religious support perceived by the patient.

Shared treatment approach: treatment received at one or two facilities.

Migration: need to migrate to receive treatment.

Previous comorbidity: chronic condition not related to cancer at the time of diagnosis.

Acquired comorbidity: chronic condition acquired due to cancer and/or cancer treatment

TABLE 3. Adherence and beliefs. $N=194$

\begin{tabular}{lcccc}
\hline & General & $\begin{array}{c}\text { Non-adherent } \\
\mathbf{4 8} \mathbf{( 2 5} \boldsymbol{\%})\end{array}$ & $\begin{array}{c}\text { Adherent } \\
\mathbf{1 4 6} \mathbf{( 7 5} \boldsymbol{\%})\end{array}$ & $\begin{array}{c}\text { Difference between adherent } \\
\text { and non-adherent patients. } \\
\boldsymbol{p} \text { value }\end{array}$ \\
\hline $\begin{array}{l}\text { Overuse score } \mathrm{X} \pm \mathrm{SD} \\
\text { Median }\end{array}$ & $3.05 \pm 0.84$ & $2.99 \pm 0.87$ & $3.25 \pm 0.71$ & 0.06 \\
Harm score $\mathrm{X} \pm \mathrm{SD}$ & 2.7 & 2.3 & 2.7 & 0.21 \\
Median & $2.23 \pm 0.69$ & $2.20 \pm 0.69$ & $2.33 \pm 0.68$ & 0.34 \\
Necessity score $\mathrm{X} \pm \mathrm{SD}$ & 1.8 & 1.8 & 3.8 & $0.13 \pm 0.73$ \\
Median & $3.84 \pm 0.68$ & $3.86 \pm 0.66$ & 3.3 & 0.13 \\
Concern score $\mathrm{X} \pm \mathrm{SD}$ & 3.4 & 3.4 & $2.90 \pm 0.79$ & 2.3 \\
Median & $2.76 \pm 0.84$ & $2.72 \pm 0.86$ & 2 & \\
\hline
\end{tabular}

$\mathrm{N}$ refers to the information provided by parents.

$\mathrm{X}=$ average value. $\mathrm{SD}=$ standard deviation .

Minimum score: 1 = strongly disagree. Maximum score: 5 = strongly agree. 
and a weekly agent and showed an intermediate level of adherence. In addition, the total treatment duration for AML was shorter than that for the other three diagnoses.

Although self-reporting questionnaires may overvalue adherence, ${ }^{14}$ in this study, the information provided by parents and patients evidenced higher adherence problems than that estimated by treating physicians without using any specific tool. The questionnaires helped to identify that the main reason for non-adherence, forgetfulness, was involuntary. The SMAQ showed a higher comparative sensitivity in relation to other self-reporting tools about adherence. ${ }^{15}$

Although the main caregiver's level of education was one of the most commonly described barriers in the bibliography, ${ }^{2,16}$ it was not associated with adherence in this sample. No difference was made between complete and incomplete secondary education, and half of sample subjects were in the secondary education group. This was probably not the most adequate categorization to evidence potential differences in adherence behavior.

Barriers in compliance were children's resistance and difficulties accessing medication. Both barriers had a strong cultural (rearing method) and political (health care system) component specific to the study population. Hence, the importance of asking about such difficulties and helping parents to improve their children's cooperation if necessary. In addition, this reveals the need to improve patient access to treatment resources.
In this sample, the subgroup of patients who agreed with the necessity for medication predominated, and that with a low perception of necessity was particularly small. These subgroups did not show differences in treatment adherence, probably because the level of concern across the sample was low. It is expected that adherence is the result of a "cost-benefit" balance between the level of necessity (high in our sample) and the concern caused by taking the medication (low in our sample). ${ }^{7}$ The most representative concern among patients was related to ignorance ("The medication is a mystery to $\mathrm{me}^{\prime \prime}$ ) and potential long-term effects. Such concerns may be solved with an adequate communication during office visits.

The younger the patient, the higher the adults' responsibility for medication administration. Autonomy in relation to treatment is progressively acquired as children grow. Adolescents usually have trouble understanding the consequences of their actions: they often believe they are invincible, get angry at the limitations imposed by disease and its management, are annoyed because they feel different from their peers, and challenge adults; all these attitudes are a threat to regular adherence. ${ }^{3,13}$ The adolescents in this sample referred more oral medication adverse events than their parents and reported a $10 \%$ lower adherence than those who did not experience symptoms. Those who mentioned new problems due to taking the medication described a $30 \%$ lower adherence. Fourteen percent of adolescents stated that it was hard for them to understand

Figure 2. Parents' and adolescents' beliefs. Parents, $N=194$. Adolescents, $N=29$

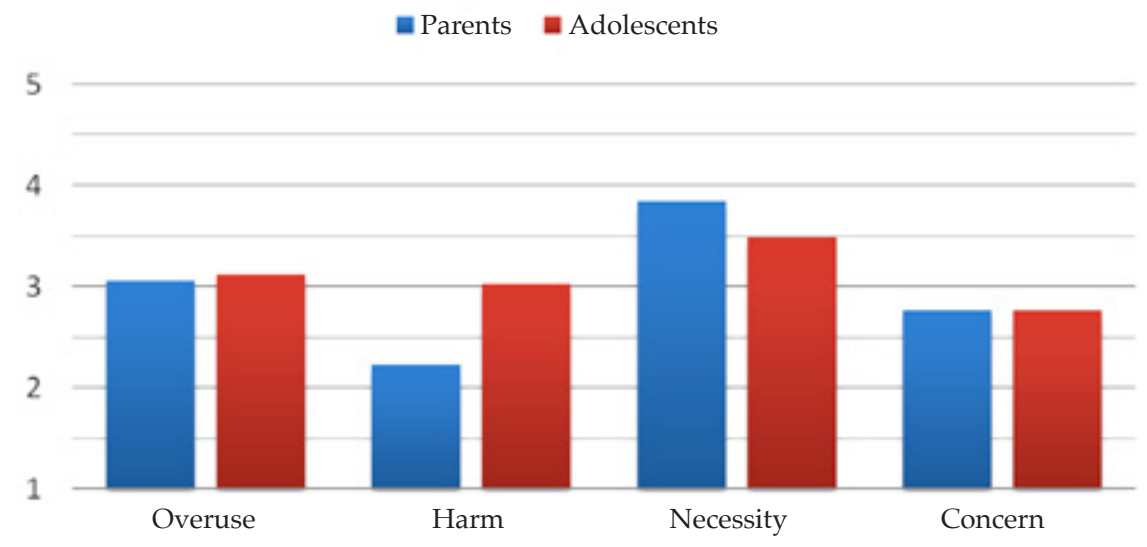

Minimum score: 1 = strongly disagree. Maximum score: 5 = strongly agree. 
medical indications.

Adolescents require supervision and support by their parents so that they comply with treatment adequately. In relation to the health care team, they should consider the convenience of adapting communication to patients' understanding capacity, convince them of the importance of maintenance in healing chances and the risks of an irregular compliance, provide any information they request, and discuss treatment aspects that concern them.

The factors that may have favored subject adherence in this sample included the existence, in almost all cases, of a stable mentor or "main caregiver" (which reduced error probabilities), a high patient incentive to comply with their regimen (given the high level of perceived necessity), and regular treatment supervision by the medical team (all patients attended scheduled regular hospital controls every one, two or three weeks).

One of the limitations of this study was that adolescents were not asked to clarify if they or their parents were partially or completely in charge of administering the medication, so the information they provided about adherence was inaccurate. Some outcome measures that the bibliography mentioned as barriers in adherence (parents' cognitive ability, strategies for treatment compliance, and the relationship between parents and patients and the health care team) were not assessed in this study. In addition, it did not allow to establish whether adherent patients administered the correct medication dose or made any mistake.

Among the study strengths, it is worth noting that the sample was numerically high and from three out of the four Argentine regions that provided pediatric cancer treatment: Northeast (Misiones), Northwest (Tucumán, Santiago del Estero), and Pampa (Córdoba, Buenos Aires) regions. Being a national facility, Hospital Garrahan provided information about patients from across the country.

The maintenance period is a critical component of cancer treatment and, if patient adherence is not optimal, healing chances are reduced. Knowing the factors associated with adherence allows to design strategies aimed at optimizing treatment, reducing barriers, and increasing its effectiveness. Some may be influenced by the health care team actions.

\section{CONCLUSIONS}

In this sample, parent-reported adherence was $75 \%$ and accounted for an intermediate value compared to that reported in other countries for the same diagnoses. Parents and patients perceived antineoplastic agents were necessary and expressed their concerns about treatment.

\section{Acknowledgments:}

We would like to thank Marisa Felice, M.D., and Susana Rodríguez, M.D.

\section{REFERENCES}

1. Moreno F. Registro oncopediátrico hospitalario argentino: incidencia 2000-2013, supervivencia 2000-2009, tendencia temporal de incidencia 2000-2013. Ciudad Autónoma de Buenos Aires: Instituto Nacional del Cáncer;2015. [Accessed on: August 3 $\left.{ }^{\text {rd }}, 2020\right]$. Available at: http: / / www.msal. gob.ar/images/stories/bes/ graficos/0000000730cnt-62publicacion-roha.pdf.

2. Bhatia S, Landier W, Shangguan M, Hageman L, et al. Nonadherence to Oral Mercaptopurine and Risk of Relapse in Hispanic and Non-Hispanic White Children With Acute Lymphoblastic Leukemia: A Report From the Children's Oncology Group. J Clin Oncol. 2012; 30(17):2094-101.

3. El Malla H, Helm N, Wilderäng U, Elbora Y, et al. Adherence tomedication: Anation-widestudy from theChildren's Cancer Hospital, Egypt. World J Psychiatry. 2013; 3(2):25-33.

4. Slone J, Chunda-Liyoka C, Perez M, Mutalima N, et al. Pediatric Malignancies, Treatment Outcomes and Abandonment of Pediatric Cancer Treatment in Zambia. PloS One. 2014; 9(2):e89102.

5. Horne R, Weinman J, Hankins M. The beliefs about medicines questionnaire: The development and evaluation of a new method for assessing the cognitive representation of medication. Int I Clin Health Psychol. 1999; 14(1):1-24.

6. Partridge AH, Archer L, Kornblith AB, Gralow J, et al. Adherence and persistence with oral adjuvant chemotherapy in older women with early-stage breast cancer in CALGB 49907: adherence companion Study 60104. J Clin Oncol. 2010; 28(14):2418-22.

7. Barillet M, Prevost V, Joly F. Clarisse B. Oral antineoplastic agents: how do we care about adherence? Br JClin Pharmacol. 2015; 80(6):1289-302.

8. Tebbi CK. Treatment compliance in childhood and adolescence. Cancer. 1993; 71(10Suppl):3441-9.

9. Rohan J, Fukuda T, Alderfer M, Wetherington Donewar $C$, et al. Measuring Medication Adherence in Pediatric Cancer: An Approach to Validation. J Pediatr Psychol. 2017; 42(2):232-44.

10. Hazarika M, Mishra R, Saikia B, Bhuyan C, et al. Causes of Treatment Abandonment of Pediatric Cancer Patients - Experience in a Regional Cancer Centre in North East India. Asian Pac J Cancer Prev. 2019; 20(4):1133-7.

11. Butow P, Palmer S, Pai A, Goodenough B, et al. Review of adherence-related issues in adolescents and young adults with cancer. J Clin Oncol. 2010; 28(32):4800-9.

12. Kennard B, Stewart S, Olvera R, Bawdon R, et al. Non adherence in adolescent oncology patients: preliminary data on psychological risk factors and relationships to outcome. J Clin Psychol Med Settings. 2004; 11(1):31-9.

13. Kondryn HJ, Edmonson CL, Hill J, Eden TO. Treatment non-adherence in teenage and young adult patients with cancer. Lancet Oncol. 2011; 12(1):100-8.

14. Ruddy K, Mayer E, Partridge A. Patient adherence and persistence with oral anticancer treatment. CA Cancer J Clin. 2009; 59(1):56-66.

15. Ortega Suárez FJ, Sánchez Plumed J, Pérez Valentín MA, Pereira Palomo $P$, et al Validación del cuestionario simplificado de adherencia a la medicación (SMAQ) en pacientes con trasplante renal en terapia con tacrolimus. Nefrología. 2011; 31(6):690-6.

16. Landier W, Hughes C, Calvillo E, Anderson N, et al. A grounded theory of the process of adherence to oral chemotherapy in Hispanic and Caucasian children and adolescents with acute lymphoblastic leukemia. J Pediatr Oncol Nurs. 2011; 28(4):203-23. 